\title{
El Carbono Acumulado en la Masa Arbórea de la Reserva Biológica Uyuca, Honduras
}

\author{
José Manuel Mora', Nelson Saúl Ulloa², Banny Omar Quezada ${ }^{3}$, Lucía Isabel López ${ }^{4}$
}

Resumen. El dióxido de carbono $\left(\mathrm{CO}_{2}\right)$ es uno de los principales gases de efecto invernadero. La quema de combustibles fósiles y la eliminación de los bosques provocan un aumento de este gas a nivel global. Los bosques contribuyen al balance global del carbono por lo que desde la convención de Kioto estos han cobrado relevancia en su papel en el ciclo del carbono. Debido a ello se ha promovido la cooperación internacional, iniciativas y proyectos con el fin de mantener niveles globales de biomasa boscosa y disminuir las tasas regionales de decline, objetivo central del proyecto REDD+. La Reserva Biológica Uyuca (RBU) en Honduras provee varios servicios ecosistémicos que no han sido cuantificados ni valorados, como el carbono acumulado en ella. La valoración es importante para la justificación de su mantenimiento y manejo. Para ayudar a solventar este vacío estimamos la cantidad de carbono acumulado que existe en la biomasa forestal de la RBU. Estratificamos la cobertura forestal y estimamos la biomasa en cada estrato. Calculamos el carbón acumulado en la reserva según la metodología del IPCC. Estimamos una biomasa aérea de 445.6 toneladas (t) por hectárea en el bosque mixto y $212.1 \mathrm{t} / \mathrm{ha}$ en el bosque de pino. La biomasa aérea en las 371 ha de bosque mixto es de 165,326 t y de 94,582 en las 446 ha de bosque de pino, para un total de 259,909 t para la RBU. La biomasa de raíces estimada fue de 58,594 t para 318,504 t de biomasa total. Esta biomasa contiene 159,252 t de carbono acumulado solo en el componente arbóreo (árboles mayores a $10 \mathrm{~cm}$ de diámetro a la altura del pecho). No tomamos en cuenta otros elementos como hierbas y arbustos. La RBU y otras áreas boscosas del país son claves en el balance del carbono local y regional y pueden ser elementos importantes para programas y proyectos basados en el protocolo de Kioto. Es necesario cuantificar el carbono acumulado en estas áreas y los recursos forestales, especialmente en el contexto de la implementación de REDD+.

Palabras clave. Bosque de pino, bosque mixto, cambio climático global, estimación de biomasa, recursos forestales, REDD+.

\section{Carbon Accumulated in the Tree Mass of the Uyuca Biological Reserve, Honduras}

\begin{abstract}
Carbon dioxide $\left(\mathrm{CO}_{2}\right)$ is a major greenhouse gas. Burning fossil fuels, clearing of forests and other factors have caused a global increase in concentration of this gas. Forests contribute to the global carbon balance and as purported in the Kyoto convention, these agroecosystems have become important in terms of their role in the carbon cycle. As a result international cooperation, initiatives and projects have been promoted to maintain the overall levels of woody biomass within forests and reduce regional rates of $\mathrm{CO}_{2}$, which is the central objective of the REDD+ project. The Uyuca Biological Reserve (UBR) in Honduras serves to this purpose but its contribution to managing the carbon cycle balance has not been quantified. Quantification of these contributions are important to justify its maintenance and management. To address this gap in knowledge, we estimated the amount of carbon stored in the UBR as tree biomass. We estimated forest strata and tree biomass in each stratum. We calculated the carbon accumulated in UBR by an IPCC recommended methodology. We estimated a biomass of 445.6 tons (t) per hectare for the mixed forest and $212.1 \mathrm{t} /$ ha for the pine forest. The aerial biomass in the 371 ha of mixed forest is $165,326 \mathrm{t}$ and $94,582 \mathrm{t}$ in the 446 ha of pine forest for a total of $259,909 \mathrm{t}$ in the reserve. The estimated root biomass was $58,594 \mathrm{t}$ for a total of $318,504 \mathrm{t}$ in UBR. This biomass contains 159,252 $t$ of carbon accumulated just in the tree component (trees larger than $10 \mathrm{~cm}$ diameter at breast high). We did not take in account other elements such as grasses and shrubs, and therefore these estimates are an underestimate of the real values. The

\footnotetext{
${ }^{1}$ Instituto Internacional en Conservación y Manejo de Vida Silvestre (ICOMVIS), Universidad Nacional (UNA), Heredia, Costa Rica. Correo electrónico josemora07@gmail.com

${ }^{2}$ Oficina Coordinadora de Proyectos, Tegucigalpa, M.D.C., Bulevar Morazán, Mall El Dorado, 5to. Nivel, Honduras. Correo electrónico nulloa@miambiente.gob.hn

${ }^{3}$ Colonia Lomas de Toncontín, Tegucigalpa, M.D.C., Honduras. Correo electrónico banyq@yahoo.com

${ }^{4}$ Cinco Esquinas de Carrizal de Alajuela, Costa Rica. Correo electrónico luciaisa2@gmail.com
} 
RBU and other woody areas in the country are key elements to ensure proper carbon cycle balance at local and regional level and can be important for programs and projects based on the Kyoto protocol. It is important to quantify the carbon accumulated in these areas and forest resources in general, especially in the context of REDD+ implementation.

Key words. Biomass estimation, forest resources, global climate change, mix forest, pine forest, REDD+.

\section{Introducción}

La concentración de gases de efecto invernadero (GEI) en la atmósfera ha aumentado considerablemente en las últimas décadas. El dióxido de carbono $\left(\mathrm{CO}_{2}\right)$ es el principal GEI y el uso de los combustibles fósiles y el cambio en el uso del suelo son considerados las dos principales fuentes netas de este gas. Una de las consecuencias de la concentración de GEl es el incremento en la temperatura ambiental de la tierra que está generando un cambio climático global (CCG). Este fenómeno se ha intensificado debido al aumento de las emisiones de $\mathrm{CO}_{2}$ producto del consumo de hidrocarburos (IPCC 2014). El ciclo del carbono está fuera de equilibrio como resultado del aumento de la combustión de combustibles fósiles por los países ricos y la eliminación de los bosques en los países tropicales (Grace et al. 2014). A la vez, el CCG está causando alteraciones en los ciclos hídricos, sequías, inundaciones y otros desastres naturales que comprometen la existencia de la vida en el planeta. Los procesos geológicos como la erosión y la actividad volcánica también contribuyen al intercambio de $\mathrm{CO}_{2}$ entre la tierra y la atmósfera, pero su contribución es importante sólo en una escala de tiempo geológico (Berner 2003).

Las discusiones sobre la limitación de las emisiones antropogénicas de $\mathrm{CO}_{2}$ a menudo se centran en la transición a fuentes de energía renovables y la captura y almacenamiento (fijación) de carbono (Ni et al. 2016). El Protocolo de Kioto y las subsecuentes Conferencias de las Partes (COP) de la Convención de Cambio Climático, han despertado el interés sobre el potencial de los ecosistemas boscosos y plantaciones forestales para fijar carbono (Magnussen y Carillo Negrete 2015). De esta manera, los bosques adquieren su protagonismo dentro de los acuerdos internacionales sobre el cambio climático y las emisiones de los GEI (Köhl et al. 2009).

Los bosques tienen un potencial significativo para la captura y almacenamiento de carbono a escala global (Ni et al. 2016). Estos son sistemas naturales complejos que contribuyen a mitigar el cambio climático global al almacenar carbono en la vegetación y en el suelo, e intercambiar carbono con la atmósfera a través de los procesos de fotosíntesis y respiración (Gasparri y Manghi 2004). Considerar este servicio ambiental y evaluarlo en términos ecológicos y forestales, podría estimular proyectos forestales dentro del marco del Mecanismo de Desarrollo Limpio. Además, en el futuro cercano necesitaremos medir este y otros aspectos para evaluar los recursos forestales en áreas grandes, especialmente en el contexto de la implementación del Proyecto "Reduced emissions from deforestation and forest degradation" conocido como REDD+ (Hayashi et al. 2015).

El Protocolo de Kyoto abrió la posibilidad de un nuevo tipo de inversiones en el sector forestal que requieren el desarrollo de indicadores de biomasa y contenido de carbono además de los parámetros clásicos necesarios para la ordenación de los bosques como el volumen (Gasparri y Manghi 2004). Los estudios de biomasa son importantes para comprender el ecosistema forestal porque permiten evaluar los efectos de una intervención con respecto al equilibrio del ecosistema (Teller 1988, Gasparri y Manghi 2004). En este contexto, es importante cuantificar el carbono acumulado en los bosques secundarios, las plantaciones forestales, los sistemas agroforestales y las áreas protegidas. El imperativo de mantener niveles globales de biomasa boscosa y disminuir las tasas regionales de decline (Hansen et al. 2010) ha promovido la cooperación internacional, iniciativas y proyectos con este fin (Plugge et al. 2011).

La generación de metodologías para estimar la fijación potencial de carbono es necesaria para los procesos de valoración económica, la definición de la línea base, la certificación y el monitoreo en proyectos de venta de certificados de reducción de emisiones de carbono. Los árboles tienen la capacidad de captar el carbono en la biomasa aérea y en la radicular. La biomasa forestal viva sobre el suelo (AGB por sus siglas en inglés) constituye la mayor contribución para 
Mora et al:: El Carbono Acumulado en la Masa Arbórea de la Reserva Biológica Uyuca, Honduras

el balance de carbono forestal (Magnussen y Carillo Negrete 2015). Por lo tanto, la evaluación se desarrolla por medio de un inventario de la biomasa total existente en el sistema y se expresan los valores en toneladas de carbono por hectárea. Para ello se utiliza la fracción de carbono que existe en la biomasa mediante métodos directos o indirectos (Russo 2009).

El método directo consiste en cortar el árbol y pesar la biomasa directamente para determinar luego su peso seco. La biomasa se puede estimar de manera indirecta a través de ecuaciones y modelos matemáticos calculados por medio de análisis de regresión entre las variables colectadas en el campo y en inventarios forestales (Brown 1997). Otra manera de estimar la biomasa es mediante el volumen del fuste y su densidad básica para determinar el peso seco y un factor de expansión para determinar la biomasa total del árbol (peso seco total). La mayor proporción de carbono se encuentra en el tallo, por lo que puede ser usado como una posible medida de almacenamiento (Sylvester y Avalos 2013). La estimación de la biomasa es ampliamente utilizada como base para calcular el carbono fijado por las plantas (Álvarez Vergnani 2015).

Aunque se ha probado que las medidas de campo tradicionales basadas en parcelas para estimar la AGB de los bosques son los más precisos, son costosos y difíciles de implementar en áreas grandes de bosque tropical denso (Thapa et al. 2015). Sin embargo, estos métodos son una buena alternativa en áreas pequeñas. Aun así, la estimación del total de carbono almacenado por los bosques tropicales tiene todavía errores significativos (Álvarez Vergnani 2015). El mayor problema es no considerar la alta variedad de formas de vida que no se toma en cuenta en los inventarios de carbono (Houghton 2005; Lima et al. 2012).

En el caso de alteraciones, los bosques liberan el carbono (Gasparri y Manghi 2004). La mayor causa de alteración de los bosques es la deforestación por lo que la protección de los hábitats boscosos es fundamental. En la actualidad, existen 3.85 mil millones de hectáreas de bosque en el mundo, $26 \%$ de la superficie terrestre, pero esta área decrece 13 millones de hectáreas por año (Thapa et al. 2015). El paradigma actual de la conservación de los bosques y de la biodiversidad en general son las áreas protegidas. El sistema de áreas protegidas de Honduras incluye varias reservas pequeñas como la Reserva Biológica Uyuca (RBU) en las las cercanías de Tegucigalpa, la capital del país. A pesar de su pequeño tamaño, la RBU presta servicios ecosistémicos de gran valor como la provisión de agua a las comunidades aledañas (Mora y López 2011). No se ha cuantificado el valor de la RBU en términos de la provisión de otros servicios ecosistémicos. Realizamos un proyecto piloto para la cuantificación de la AGB y el carbono almacenado en la masa forestal de la RBU para ayudar a comenzar a llenar estos vacíos.

\section{Materiales y Métodos}

El estudio fue en la Reserva Biológica Uyuca $\left(14^{\circ} 00^{\prime} 11^{\prime \prime}\right.$ y $14^{\circ} 01^{\prime} 40^{\prime \prime} \mathrm{N}, 87^{\circ} 01^{\prime} 20^{\prime \prime}$ y $\left.87^{\circ} 05^{\prime} 00^{\prime \prime} \mathrm{W}\right)$, departamento de Francisco Morazán, Honduras (Mora et al. 2013). La RBU consta de la zona núcleo de 237.1 ha, 52.0 de ellas cubiertas con bosque nublado latifoliado y la zona de amortiguamiento de 579.8 ha (Mora et al. 2013) para un total de 816.9 ha. El núcleo es el área superior del cerro Uyuca comprendido entre la cota de $1,700 \mathrm{msnm}$ y el punto más alto a 2008 msnm (Mora et al. 2013). La temperatura media anual fue entre 14.8 y $16.6{ }^{\circ} \mathrm{C}$, basado en un gradiente térmico de $6^{\circ} \mathrm{C}$ de descenso en la temperatura por cada $1,000 \mathrm{~m}$ de ascenso sobre el nivel del mar. La precipitación promedio anual fue de $2,000 \mathrm{~mm} 0$ un poco más, con fundamento en la fisonomía de la vegetación natural madura (Agudelo et al. 2012). Ecológicamente el área corresponde a la zona de vida del bosque muy húmedo montano bajo subtropical (Mora et al. 2013).

En la RBU se pueden diferenciar tres tipos de comunidades vegetales, el bosque latifoliado maduro, los bosques mixtos y los relativamente puros dominados por pinabete (Pinus maximinol), además de bosques secundarios en diferentes fases de sucesión. En el bosque latifoliado existe una gran variedad y riqueza de especies de bromelias y epífitas que constituyen hábitats de importancia para otras especies de flora y fauna (Mora et al. 2013). Aproximadamente el $50 \%$ de la estructura de la comunidad florística en la zona núcleo de la RBU está determinada por cinco especies: Persea americana var nubigena, Querqus benthamii, Ardisia venosa, $Q$. bumelioides y Miconia theaezans (Mora et al. 2013).

Existen varias metodologías aplicables para la medición de la cantidad de carbono en la biomasa forestal (Russo 2009); una muy conocida es la publicada por FAO (Brown 1997, FAO 2001) y otra universalmente aceptada es la descrita por el Panel 
Intergubernamental sobre el Cambio Climático (IPCC 2003, 2006), en sus directrices para los inventarios nacionales de gases de efecto invernadero (Russo 2009). Hay que señalar que las ecuaciones alométricas usadas para convertir el diámetro y la altura de los árboles a biomasa introducen incertidumbre, especialmente para los árboles grandes que son los que normalmente contienen más carbono (Chave et al. 2005).

Como primer paso estratificamos el bosque de la RBU mediante fotografías aéreas y verificación de campo para establecer los tipos de bosque y su extensión existentes en el área de estudio. Determinamos dos tipos de bosque o estratos, el bosque nublado o mixto (incluye el bosque latifoliado) y el bosque de pino. Realizamos un levantamiento de cinco parcelas circulares de $500 \mathrm{~m}^{2}$ por cada estrato identificado (10 en total $=5,000 \mathrm{~m}^{2}$ ) y medimos el total de fustes con un DAP igual o mayor a $10 \mathrm{~cm}$. La intensidad de muestreo fue de solamente $0.06 \%$ la cual, aunque muy baja, depara resultados importantes para las estimaciones pretendidas. Además medimos con un clinómetro la altura de cada uno de los árboles dentro de la parcela. Cubicamos los datos obtenidos mediante las siguientes fórmulas:

Donde:

$$
A B=D^{2} \times 0.7854
$$

$\mathrm{AB}=$ Área Basal en metros cuadrados

$\mathrm{D}=$ Diámetro a la altura del pecho (aproximado a 1.30

m) en metros

Donde:

$$
V_{t}=A B \times H \times f f
$$

$V_{t}=$ Volumen total

$\mathrm{AB}=$ Área Basal

$\mathrm{H}=$ Altura total

$\mathrm{ff}=$ Factor de forma

El factor de forma considerado equivale a 0.5 , es decir la mitad del volumen de un cilindro de diámetro y altura correspondientes. Para la estimación del volumen para el pino se pudo usar una regresión volumétrica. Existen otras metodologías de mayor precisión, sin embargo, al ser esta una estimación básica general como proyecto piloto para estimular a estudios más avanzados, se prefirió utilizar las estimaciones más básicas que a la vez son más conservadoras.

La biomasa arbórea la calculamos mediante la consideración de los factores aprobados por el IPCC en sus normas para el cálculo de carbono en los países (IPCC 2006). La biomasa del volumen (BV) es el producto de la multiplicación del $\mathrm{V}_{t}$ por la densidad que corresponde a 0.6 (Brown 1997). La densidad es la masa de la madera seca al horno por unidad de volumen (Gasparri y Manghi 2004). Esta es el resultado del factor peso seco/volumen de la madera en gramos por centímetros cúbicos, cuyo valor ha sido promediado entre las especies por estrato y estimado por el IPCC. Una valoración más precisa se lograría con la medición de la densidad de cada especie con muestras individuales. Sin embargo, no hicimos identificaciones individuales específicas de los componentes del estrato arbóreo de Uyuca. No obstante, esta aproximación general según los datos del IPCC, es suficiente para los propósitos de este estudio.

Al valor estimado de la BV se le agrega el factor de expansión de biomasa (FEB) que corresponde a 1.7 en el caso del bosque mixto y de 2.2 en el caso del bosque de pino (según el límite de 190 t/ha de $V_{t}$, Brown 1997). El FEB es un factor calculado para inferir la biomasa de un árbol o de un bosque a partir de medidas dasométricas tales como el diámetro a la altura del pecho (DAP) o el volumen total $\left(\mathrm{V}_{\mathrm{t}}\right)$. Generalmente, el FEB representa un $60 \%$ adicional debido al contenido en ramas y follaje (Brown 1997).

El valor de la biomasa aérea calculada (BV) lo multiplicamos por el área total para obtener la biomasa aérea total del estrato boscoso correspondiente. Este valor lo multiplicamos por un factor determinado para estimar la biomasa de las raíces. Este valor es de 0.24 para el bosque nublado o mixto y de 0.20 para el bosque de pino. La suma de los dos productos (biomasa aérea total más la biomasa de raíces) da como resultado la biomasa total del estrato boscoso. Para estimar la cantidad total de carbono almacenado en la biomasa arbórea de la RBU utilizamos el factor aprobado por el IPCC de $50 \%$ del peso de la biomasa existente (Brown 1997). Se calcula que con este protocolo se está sobrestimando un $3.7 \%$ de $\mathrm{C}$ en la madera de los bosques tropicales que tienen pocos o no tienen compontes de coníferas (Thomas y Martin 2012). En la RBU, los dos estratos boscosos son compuestos de mayoritariamente coníferas 0 son mixtos. Aun así estimamos un ámbito de $\pm 5 \%$ del $C$ calculado para incluir una posible variación.

Hay que tomar en cuenta que solamente calculamos el volumen de la biomasa de los árboles. No incluimos la biomasa existente en los arbustos, las 
hierbas, la hojarasca y la materia orgánica o humus incorporado al suelo ni de ningún otro elemento adicional a los árboles con más de $10 \mathrm{~cm}$ de DAP.

\section{Resultados}

Determinamos que el bosque nublado ocupa un área de 371 ha en la RBU mientras que el bosque de pino cubre 446 ha (Cuadro 1). El volumen del bosque nublado (mixto) de la RBU fue de $426.8 \mathrm{~m}^{3} / \mathrm{ha}$ y el del bosque de pino fue de $159.9 \mathrm{~m}^{3} / \mathrm{ha}$ (Cuadro 1). Como resultado, la biomasa del volumen inventariado (BV) fue mayor para el bosque nublado (256.1 t/ha, cuadro 1). De igual manera la biomasa aérea por hectárea del bosque nublado fue mayor que la del bosque de pino (Cuadro 1).

La biomasa del bosque mixto es de 205,005 toneladas (t) y la del bosque de pino es de 113,498 $t$ (Cuadro 2). Así, el total de biomasa de los árboles de la RBU es de 318,503 t (Cuadro 2). Como resultado el total de carbono acumulado es de 159,252 t en los dos estratos boscoso de la RBU (Cuadro 2).

El carbono contenido en los árboles con un DAP mayor a $10 \mathrm{~cm}$ puede variar entre en un $5 \%$ con respecto al valor estimado (Cuadro 3). Esto según estimaciones hechas por el IPCC (2006).

Cuadro 1. Biomasa aérea (BA) en toneladas por hectárea ( $t / h a)$ basada en la biomasa del volumen inventariado $(B V)$ según el volumen determinado $\left(\mathrm{V}_{\mathrm{t}}\right)$ por hectárea para los dos estratos boscosos de la Reserva Biológica Uyuca, Honduras.

\begin{tabular}{lcrrc}
\hline Estrato boscoso & BA t/ha & BV/ha & V $/$ ha & Área total (ha) \\
\hline Bosque nublado & 445.6 & 256.1 & 426.8 & 371 \\
Bosque de pino & 212.1 & 95.9 & 159.9 & 446 \\
\hline & $\overline{\mathbf{X}}=\mathbf{3 2 8 . 8}$ & $\overline{\mathbf{X}}=\mathbf{1 7 6 . 0}$ & $\overline{\mathbf{X}}=\mathbf{2 9 3 . 3}$ & Total = 817 \\
\hline
\end{tabular}

Cuadro 2. Biomasa total y carbono por estrato boscoso y total en toneladas estimado para la Reserva Biológica Uyuca, Honduras.

\begin{tabular}{lcccc}
\hline Estrato & Biomasa aérea & Biomasa raíces & Biomasa total & Total de carbono \\
\hline Bosque nublado & 165,326 & 39,678 & 205,005 & 102,503 \\
Bosque de pino & 94,582 & 18,916 & 113,498 & 56,749 \\
\hline Total & $\mathbf{2 5 9 , 9 0 8}$ & $\mathbf{5 8 , 5 9 4}$ & $\mathbf{3 1 8 , 5 0 3}$ & $\mathbf{1 5 9 , 2 5 2}$ \\
\hline
\end{tabular}

Cuadro 3. Estimación de la variación del contenido de carbono, en toneladas, en los árboles con un diámetro a la altura del pecho (DAP) mayor a $10 \mathrm{~cm}$ en la Reserva Biológica Uyuca, Honduras.

\begin{tabular}{lccc}
\hline Estrato & Total de Carbono & Límite 5\% inferior & Límite 5\% superior \\
\hline Bosque nublado & 102,503 & 97,378 & 107,628 \\
Bosque de pino & 56,749 & 53,912 & 59,586 \\
\hline Total & $\mathbf{1 5 9 , 2 5 2}$ & $\mathbf{1 5 1 , 2 8 9}$ & $\mathbf{1 6 7 , 2 1 4}$ \\
\hline
\end{tabular}




\section{Discusión}

La estimación de la biomasa seca en los bosques es de gran interés para estudios de flujos de nutrientes en los ecosistemas y, más recientemente, para estudios de cuantificación del carbono y su relación con el cambio climático. La Convención de las Naciones Unidas sobre el Cambio Climático y su Protocolo de Kyoto reconocen el papel de los bosques en la mitigación del cambio climático (Pilli et al. 2016). Desde la celebración de la reunión de Kyoto, se comenzó a percibir a los bosques como productores de servicios ambientales para la sociedad, más allá de la concepción clásica de la producción de madera (Gasparri y Manghi 2004). La fijación de $\mathrm{CO}_{2}$ en la madera mediante la fotosíntesis hace a los bosques fundamentales en el balance mundial de este gas en la atmósfera (Magnussen y Carillo Negrete 2015).

Los bosques son importantes porque secuestran gran cantidad de carbono atmosférico por ser componentes clave del ciclo global del carbono y ayudan a mitigar el calentamiento global (Hayashi et al. 2015). Las aproximadamente 160,000 toneladas de carbono acumulado en la masa boscosa de la RBU (Cuadros 2 y 3 ) representan un servicio ecosistémico no valorado que se debe sumar a otros bienes y servicios más tangibles como el agua que provee la RBU. Estos dos servicios ecosistémicos justifican plenamente su mantenimiento y evitar a toda costa su afectación por deforestación u otras formas de deterioro del hábitat. Esto es especialmente primordial bajo el contexto de la implementación de REDD+. La REDD+ requiere la capacidad de medir el carbono acumulado y los cambios en la cubierta forestal para cada tipo de bosque. Estos datos se utilizan para estimar las emisiones de $\mathrm{CO}_{2}$ procedentes de la deforestación y la degradación forestal ((Hayashi et al. 2015). La RBU, además, protege elementos significativos de la biodiversidad (Mora y López 2011).

El manejo de la adaptación reduciría los riesgos del cambio climático para los ecosistemas del mundo (Thomas et al. 2016). Reducir la deforestación y la degradación del bosque contribuye a los esfuerzos de mitigación del cambio climático global (Bustamante et al. 2016). El carbono acumulado en la RBU (Cuadros 2 y 3 ) es una contribución significativa para el balance del $\mathrm{CO}_{2}$ atmosférico en una zona afectada por la deforestación. Debido a la forma de estimación y a la variación que esta puede tener, el total de carbono puede estar sobrestimado. Esta estimación está basada en un porcentaje de carbón de $50 \%$ en los árboles según la sugerencia de la IPCC (2006). Debido a que este porcentaje puede variar, estimamos un límite inferior mínimo para la cantidad de carbono acumulado en la RBU. Este total de carbono acumulado mínimo fue de 151,289 toneladas (Cuadro 3). Tampoco tomamos un descuento de volumen por pudriciones o defectos, lo que restaría biomasa a la cantidad estimada. Aun así, la cantidad de carbono acumulado es de gran significancia ecológica y ambiental. No obstante, existe una cantidad adicional muy significativa de carbono acumulado en la RBU en otros elementos no considerados en esta evaluación (epífitos, hierbas o arbustos). La diversidad de formas de vida de los bosques tropicales dificulta estimar el papel de este ecosistema en la dinámica del carbono (Álvarez Vergnani 2015).

Estimar la cantidad de carbono en el resto de la biomasa de la RBU (adicional a la de los árboles con un DAP igual o mayor a $10 \mathrm{~cm}$ ) requiere de un estudio intensivo que incluye muestras de laboratorio obtenidas de cada uno de los aportantes no incluidos. Esto es una actividad, que aunque es muy laboriosa, queda pendiente por realizar para mostrar el valor de este bosque y ayuda en el manejo de la reserva. Aun así, la cantidad que aporta el componente arbóreo da una idea de la importancia de la RBU como sumidero de carbono, principalmente obtenido del aire en forma de bióxido de carbono $\left(\mathrm{CO}_{2}\right)$. Además, la RBU absorbe otros gases tales como los compuestos nitrogenados que también tienen efecto y participan en el cambio climático al afectar la capa de ozono.

Este trabajo es una primera aproximación para la obtención de valores relacionados con la provisión de servicios ecosistémicos de una pequeña reserva biológica como la RBU. El reconocimiento de estos servicios y valores, entre ellos la mitigación del cambio climático (Canadell y Schulze 2014), son muy importantes para la formulación de políticas y la evaluación de la situación de los bosques nativos. La estimación correcta del carbono fijado por los bosques tropicales brindaría mayores criterios para calcular pagos más justos por servicios ambientales con mayor impacto como incentivos para su conservación (Álvarez Vergnani 2015). Si bien es cierto que el mercado de carbono no ha llegado a ser una iniciativa global y los precios son bajos, existe un gran potencial en el futuro (Redmond y Convery 2015). Además, si este tipo de áreas es manejado acorde con los escenarios actuales y futuros del cambio climático, su 
Mora et al:: El Carbono Acumulado en la Masa Arbórea de la Reserva Biológica Uyuca, Honduras

valor aumenta en beneficio de los usuarios de sus servicios ecosistémicos. La RBU podría ser un ejemplo de manejo bajo el contexto de implementación de REDD+. Además, un punto clave del mercado de carbono ha sido el ofrecer nuevas oportunidades a los países en desarrollo y las comunidades de escasos recursos para lograr el desarrollo sostenible (Siedenburg et al. 2016).

La RBU es una de las primeras áreas protegidas de la región que ha incluido en su zonificación una subzona de adaptación al cambio climático (Mora et al. 2013). Esta es una de las estrategias para reducir las amenazas críticas actuales y potenciales a la RBU. El cambio climático y sus impactos asociados añaden una nueva dimensión y urgencia al reto de adaptación (Antwi-Agyeia et al. 2015). La subzona de adaptación al cambio climático (SACC) es una faja de 165 ha situada a partir de los 1,600 msnm, que es la línea de condensación de nubes en Uyuca (Agudelo et al. 2016). Esta condensación es equivalente a la cantidad de lluvia horizontal que se capta y adiciona al agua de lluvia captada por infiltración en la RBU (Mora 2014). En la SACC se podrá poner en práctica la regeneración asistida, la restauración ecológica y otras técnicas que permitan mejorar la cobertura boscosa con fines hídricos y de conservación. La precipitación en los trópicos podría decrecer mucho y tal vez debilitar la circulación atmosférica tropical (Vecchi et al. 2006). La SACC de la RBU es pionera no solo en su denominación, sino en prácticas de ecología aplicada como medidas de adaptación al cambio climático (Mora 2014).

\section{Literatura Citada}

Agudelo, N., J.M. Mora, S. Pérard y J.C. Jut Solórzano. 2012. Extensión del Bosque Nublado y su Contribución de la Lluvia Horizontal a la Precipitación Total en la Reserva Biológica Uyuca, Honduras. Ceiba 53(2):109123.

Álvarez Vergnani, C. 2015. Ecuaciones alométricas para estimar el contenido de carbono en palmas de sotobosque del corredor San Juan-La Selva, Costa Rica. Tesis de Licenciatura. Universidad de Costa Rica. $91 \mathrm{p}$.

Antwi-Agyeia, P., A.J. Dougilla y L.C. Stringera. 2015. Barriers to climate change adaptation: evidence from northeast Ghana in the context of a systematic literature review. Climate and Development 7(4):297-309.

Berner, R.A. 2003. The long-term carbon cycle, fossil fuels and atmospheric composition. Nature 426(6964):323326.
Brown, S. 1997. Estimating biomass and biomass change of tropical forests: a primer. FAO Forestry Paper - 134. Consultado 10 diciembre 2015. Disponible en: http://www.fao.org/docrep/w4095e/w4095e00.HTM

Bustamante, M., I. Roitman, T. Mitchell Aide, A. Alencar, L.O. Anderson, L. Aragao, G.P. Asner, J. Barlow, E. Berenguer, J. Chambers, M.H. Costa, T. Fanin, L. Ferreira, J. Ferreira, M. Keller, W.E. Magnuson, L. Morales-Barquero, D. Morton, J.P.H.B. Ometto, M. Palace, C.A. Peres, D. Silvério, S. Trumbore y I.C.G. Vieira. 2016. Toward an integrated monitoring framework to assess the effects of tropical forest degradation and recovery on carbon stocks and biodiversity. Global Change Biology 22:92-109.

Canadell, J.G. y E.D. Schulze. 2014. Global potential of biospheric carbon management for climate mitigation. Nature Communications 5(5282):1-12.

Chave, J., C. Andalo, S. Brown, M.A. Cairns, J.Q. Chambers, D. Eamus, H. Fölster, F. Fromard, N. Higuchi, T. Kira, J.P. Lescure, B.W. Nelson, H. Ogawa, H. Puig, B. Riéra y T. Yamakura. 2005. Tree allometry and improved estimation of carbon stocks and balance in tropical forests. Oecologia 145:87-99.

FAO (Food and Agriculture Organization of the United Nations). 2001. Global forest resources assessment 2000 - main report. FAO. 511 p.

Gasparri, I. y E. Manghi. 2004. Estimación de volumen, biomasa y contenido de carbono de las regiones forestales argentinas. Informe final. Unidad de Manejo del Sistema de Evaluación Forestal. Dirección de bosques, Secretaría de ambiente y desarrollo sustentable. Argentina. 26 p.

Grace J., E. Mitchard y E. Gloor. 2014. Perturbations in the carbon budget of the tropics. Global Change Biology 20(10):3238-3255.

Hansen M.C., S.V. Stehman, P.V. Potapov 2010. Quantification of global gross forest cover loss. Proceedings of the National Academy of Sciences 107:8650-8655.

Hayashi, M., N. Saigusa, Y. Yamagata y T. Hirano. 2015. Regional forest biomass estimation using ICESat/GLAS spaceborne LiDAR over Borneo. Carbon Management 6 $(1,2): 19-33$.

Houghton, R.A. 2005. Aboveground biomass and the global carbon balance. Global Change Biology 11(6):945-958.

IPCC (Intergovernamental Panel for Climate Change of the United Nations). 2006. Directrices del IPCC de 2006 para los inventarios nacionales de gases de efecto invernadero. Volumen 4: Agricultura, silvicultura y otros usos de la tierra. Capítulo 4: Tierras Forestales. H. Aalde, P. Gonzalez, M. Gytarsky, T. Krug, W.A. Kurz, S. Ogle, J. Raison, D. Schoene y N.H. Ravindranath (Eds). Intergovernmental Panel on Climate Change (IPCC), IPCC/OECD/IEA, París, Francia. $66 \mathrm{p}$. 
IPCC (Intergovernmental Panel for Climate Change of the United Nations). 2014. 2013 Supplement to the 2006 IPCC Guidelines for National Greenhouse Gas Inventories: Wetlands. T. Hiraishi, T. Krug, K. Tanabe, N. Srivastava, J. Baasansuren, M. Fukuda y T.G. Troxler (eds). Intergovernamental Panel for Climate Change of the United Nations, Switzerland. 354 p.

IPCC (Intergovernmental Panel on Climate Change). 2003. Good Practice Guidance for Land Use, Land-Use Change and Forestry (GPG-LULUCF). J. Penman, M. Gytarsky, T. Hiraishi, T. Krug, D. Kruger, R. Pipatti, L. Buendia, K. Miwa, T. gara, K. Tanabe y F. Wagner (Eds.). IPCC National Greenhouse Gas Inventories Programme, Hayama, Japan. 590 p.

Köhl M., T. Baldauf, D. Plugge y J. Krug. 2009. Reduced emissions from deforestation and forest degradation (REDD): a climate change mitigation strategy on a critical track. Carbon Balance Management 4:10.

Lima, J.N.A., R. Suwa, H.P.G.M. Ribeiro, T. Kajimoto, J. Santos, P.R. Silva, C.A.S. de Souza, P.C. de Barros, H. Noguchi, M. Ishizuka y N. Higuchi. 2012. Allometrics models for estimating above-and below-grownd biomass of tropical rainforest at Saõ Gabriel da Cachoeira in upper Rio Negro, Brazilian Amazon. Forest Ecology and Management 277:163-172.

Magnussen, S. y O.I. Carillo Negrete. 2015. Model errors in tree biomass estimates computed with an approximation to a missing covariance matrix Carbon Balance Management 10:21.

Mora, J.M. 2014. Manejo de la Reserva Biológica Uyuca, Honduras, en perspectiva del cambio climático global. Simposio de Cambio Climático, XVIII Congreso Regional de la SMBC, Copán, Honduras.

Mora, J.M. y L.I. López. 2011. El Manejo de la Reserva Biológica Uyuca en el Contexto Nacional y Global del Sistema de Áreas Protegidas. Ceiba 52(1):39-54.

Mora, J.M., L.I. López, M. Acosta y P. Maradiaga. Plan de Manejo Reserva Biológica Uyuca 2013-2025. Instituto Nacional de Conservación y Desarrollo Forestal, Áreas Protegidas y Vida Silvestre y Escuela Agrícola Panamericana. Honduras. $165 \mathrm{p}$.

Ni, Y., G.S. Eskeland, J. Giske y J.P. Hansen. 2016. The global potential for carbon capture and storage from forestry. Carbon Balance and Management 11(3):1-8.
Pilli, R., G. Grassi, W.A. Kurz, R.A. Viñas y N.H. Guerrero 2016. Modelling forest carbon stock changes as affected by harvest and natural disturbances. I. Comparison with countries' estimates for forest management. Carbon Balance and Management 11(5):1-18.

Plugge D., T. Baldauf, M. Köhl. 2011. Reduced emissions from deforestation and forest degradation (REDD): why a robust and transparent monitoring, reporting and verification (MRV) system is mandatory. p. 155-170. In J. Blanco y $\mathrm{H}$. Kheradmand (eds). Climate changeresearch and technology for adaptation and mitigation, chap 9. InTech, Rijeka, Croatia.

Redmond, L. y F. Convery. 2015. The global carbon marketmechanism landscape: pre and post 2020 perspectives. Climate Policy 15(5):647-669.

Russo, R.O. 2009. Guía práctica para la medición de la captura de carbono en la biomasa forestal. Unidad de Carbono Neutro, Universidad EARTH. Limón, Costa Rica. 17 p.

Siedenburg, J., S. Brown y S. Hoch. 2016. Voices from the field - carbon markets and rural poverty as seen from Madagascar and Mali. Climate and Development 8(1):10-25.

Sylvester, O. y G. Avalos. 2009. Illegal palm heart (Geonoma edulis) harvest in Costa Rican national parks: Patterns of consumption and extraction. Economic Botany 63(2):179-189.

Teller, A., 1988. Biomass, productivity and wood waste evaluation in a Spruce (Picea abies) forest (Strinchmps 983). Commonwealth Forestry Review 7(2):129-148.

Thapa, R.B., T. Motohka, M. Watanabe y M. Shimada. 2015. Time- series maps of aboveground carbon stocks in the forests of central Sumatra. Carbon Balance and Management 10(23):1-13.

Thomas, S.C. y A.R. Martin. 2012. Carbon Content of Tree Tissues: A Synthesis. Forests 3(2):332-352.

Thomas, S.M., S.W. Griffiths y S.J. Ormerod. 2106. Beyond cool: adapting upland streams for climate change using riparian woodlands Global Change Biology 22:310-324.

Vecchi, G.A., B.J., Soden, A.T. Wittenberg, I.M. Held, A. Leetmaa y M.J., Harrison. 2006. Weakening of tropical Pacific atmospheric circulation due to anthropogenic forcing. Nature 441(7089):73-76.

Recibido para publicación el 27 de mayo de 2016. Aceptado para publicación el 10 de enero de 2017. 